\title{
Co-association of methotrexate and SPIONs into anti-CD64 antibody-conjugated PLGA nanoparticles for theranostic application
}

This article was published in the following Dove Press journal:

International Journal of Nanomedicine

23 October 2014

Number of times this article has been viewed

\author{
Catarina Costa Moura ${ }^{1,2}$ \\ Marcela A Segundo' \\ José das Neves 3,4 \\ Salette Reis' \\ Bruno Sarmento 3,4
}

'REQUIMTE, Department of Chemical Sciences, Faculty of Pharmacy,

University of Porto, Porto, Portugal;

${ }^{2}$ Faculty of Engineering, University of Porto, Porto, Portugal; ${ }^{3}$ CESPU, Instituto de Investigação e Formação Avançada em Ciências e Tecnologias da Saúde, Instituto de Ciências da Saúde-Norte, Gandra PRD, Portugal; ${ }^{4}$ INEB - Instituto de Engenharia Biomédica, University of Porto, Porto, Portugal
Correspondence: Bruno Sarmento Instituto de Engenharia Biomédica, BioCarrier Group, Universidade do Porto, Rua do Campo Alegre 823, 4150-180 Porto, Portugal

Tel +35I 226074900

Fax +35I 226094567

Email bruno.sarmento@ineb.up.pt

Salette Reis

REQUIMTE, Department of Chemical Sciences, Faculty of Pharmacy, University of Porto, Rua de Jorge Viterbo Ferreira 228, 4050-3। 3 Porto, Portugal

Tel +35I 220428500

Fax +35I 226093390

Email shreis@ff.up.pt

\begin{abstract}
Background: Rheumatoid arthritis (RA) is an autoimmune disease with severe consequences for the quality of life of sufferers. Regrettably, the inflammatory process involved remains unclear, and finding successful therapies as well as new means for its early diagnosis have proved to be daunting tasks. As macrophages are strongly associated with RA inflammation, effective diagnosis and therapy may encompass the ability to target these cells. In this work, a new approach for targeted therapy and imaging of RA was developed based on the use of multifunctional polymeric nanoparticles.
\end{abstract}

Methods: Poly(lactic-co-glycolic acid) nanoparticles were prepared using a single emulsionevaporation method and comprised the co-association of superparamagnetic iron oxide nanoparticles (SPIONs) and methotrexate. The nanoparticles were further functionalized with an antibody against the macrophage-specific receptor, CD64, which is overexpressed at sites of RA. The devised nanoparticles were characterized for mean particle size, polydispersity index, zeta potential, and morphology, as well as the association of SPIONs, methotrexate, and the anti-CD64 antibody. Lastly, the cytotoxicity of the developed nanoparticles was assessed in RAW 264.7 cells using standard MTT and LDH assays.

Results: The nanoparticles had a mean diameter in the range of 130-200 nm and zeta potential values ranging from $-32 \mathrm{mV}$ to $-16 \mathrm{mV}$. Association with either methotrexate or SPIONs did not significantly affect the properties of the nanoparticles. Conjugation with the anti-CD64 antibody, in turn, caused a slight increase in size and surface charge. Transmission electron microscopy confirmed the association of SPIONs within the poly(lactic-co-glycolic acid) matrix. Both antiCD64 and methotrexate association were confirmed by Fourier transform infrared spectroscopy, and quantified yielding values as high as $36 \%$ and $79 \%$, respectively. In vitro toxicity studies confirmed the methotrexate-loaded nanosystem to be more effective than the free drug.

Conclusion: Multifunctional anti-CD64-conjugated poly(lactic-co-glycolic acid) nanoparticles for the combined delivery of methotrexate and SPIONs were successfully prepared and characterized. This nanosystem has the potential to provide a new theranostic approach for the management of RA.

Keywords: Fc $\gamma$ RI, methotrexate, poly(lactic-co-glycolic acid), superparamagnetic iron oxide nanoparticles, targeted drug delivery

\section{Introduction}

Rheumatoid arthritis (RA) is one of the most common and severe autoimmune diseases affecting the joints. This chronic inflammatory disease, in which the immune system attacks healthy tissue lining the joints, leads to functional disability and reduced quality of life, as a result of bone and cartilage destruction, joint swelling, and pain. RA is a widely prevalent systemic disease and affects $1 \%$ of the population around the globe. ${ }^{1-3}$ 
Since the RA inflammatory process remains unclear, finding effective therapies and tools for early diagnosis has been extremely challenging and remain non-existent or with limited efficacy. ${ }^{1-3}$

Diagnosis of RA can be a demanding task, considering that the disease may occur even before symptoms start to manifest themselves. Additionally, confirmation of the presence of this autoimmune disease requires use of several different criteria to establish a definite diagnosis, leading to a high risk of overtreatment. ${ }^{4}$ Magnetic resonance imaging (MRI) has been attracting considerable medical interest for early disease detection and drug therapy monitoring., 5,6 Superparamagnetic iron oxide nanoparticles (SPIONs) have emerged as highly effective contrast agents for MRI, ${ }^{6}$ but active targeting strategies are required in order to increase their accumulation at tissues of interest while decreasing nonspecific biodistribution in order to reduce background interference.

Currently, the gold standard for RA therapy is methotrexate (MTX), a drug approved by US Food and Drug Administration. ${ }^{8}$ This drug is usually administered together with other disease-modifying antirheumatic drugs, and sometimes in combination with short-term, low-dose glucocorticoids or tumor necrosis factor inhibitors. ${ }^{9}$ However, due to the lack of targeting ability using the intravenous formulations available, this therapeutic strategy does not allow specific distribution of MTX to the affected joints, and leads to drug accumulation in healthy tissues, causing harmful side effects..$^{1,3,10}$ Therefore, additional research is required in order to develop novel strategies for achieving effective and major long-term approaches for RA therapies, aiming to prevent joint destruction and associated comorbidities.

In the particular case of RA, recent studies have proposed that insufficient apoptosis of synovial inflammatory cells, especially macrophages, may contribute to persistence of the disease. Since macrophages play a pivotal role in progression of the disease, effective imaging and therapy systems may rely on the ability to target these cells. ${ }^{3}$

Bearing this in mind, a new approach for RA theranostics may take advantage of the vast potential of nanomedicine. A new wave of medical innovation is emerging due to the possibility of multifunctionalization in nanomedicine-based strategies, since nanoparticles (NPs) may have the ability to: carry therapeutic agents; be conjugated to specific ligands, namely antibodies, to target a specific tissue or organ; and amplify imaging signals, by coencapsulating contrast enhancers; among other possibilities. ${ }^{10,11}$
This study aimed to develop a nanoparticulate system that can actively target macrophages for RA imaging and therapy by intravenous administration. The work consists of the association of SPIONs as a contrast agent for MRI, and MTX for RA therapy into poly(lactic-co-glycolic acid) (PLGA) NPs. Combining these two agents in a single platform, it may be possible to simultaneously monitor and provide therapy for RA. In addition, the work comprises functionalization of PLGA NPs with a monoclonal antibody against the macrophage-specific cell surface receptor, CD64, which is overexpressed in RA. ${ }^{12}$ Different PLGA-based NPs were prepared in order to compare the effects of each component (ie, MTX, SPIONs, and anti-CD64 antibody) on the properties of the NPs.

\section{Materials and methods Materials}

Acid-terminated PLGA copolymer (50:50 Purasorb $^{\circledR}$ PDLG 5002A) was a kind gift from Purac Biomaterials (Gorinchem, the Netherlands). The MTX was obtained courtesy of Excella GmbH (Feucht, Germany). Iron oxide nanocrystals $(10 \mathrm{~nm}$, coated with oleic acid and dispersed in chloroform $25 \mathrm{mg} / \mathrm{mL}$ ), were provided by Ocean NanoTech LLC (Springdale, AR, USA). Anti-Human CD64 (Fc gamma Receptor 1) antibody solution $(1.0 \mathrm{mg} / \mathrm{mL}$ ) was purchased from eBioscience (San Diego, CA, USA). A Thermo-Scientific Coomassie Plus ${ }^{\mathrm{TM}}$ (Bradford) assay kit was sourced from Pierce Biotechnology (Rockford, IL, USA). Acetonitrile was provided by Merck KGaA (Darmstadt, Germany). The lactate dehydrogenase (LDH) cytotoxicity detection kit was from Takara Bio Inc. (Shiga, Japan). Poly(vinyl alcohol) (87\%-90\% hydrolyzed, with an average molecular weight of $30-70 \mathrm{kDa}$ ), ethyl acetate, 2-morpholinoethanesulfonic acid (MES), 1-ethyl-3-(3-dimethylaminopropyl) carbodiimide hydrochloride (EDC), N-hydroxysulfosuccinimide (NHS), sodium phosphate dibasic dihydrate, citric acid, dimethyl sulfoxide, thiazolyl blue tetrazolium bromide $98 \%$ (MTT), and Triton ${ }^{\mathrm{TM}} \mathrm{X}-100$ (for molecular biology) were purchased from Sigma-Aldrich (St Louis, MO, USA). Dulbecco's phosphate-buffered saline 10× (pH 7.4), Dulbecco's Modified Eagle's Medium (DMEM, high glucose, GlutaMAX ${ }^{\mathrm{TM}}$ supplement, pyruvate), fetal bovine serum, penicillin-streptomycin $(10,000 \mathrm{U} / \mathrm{mL})$ and Fungizone $^{\circledR}$ antimycotic were purchased from Gibco ${ }^{\circledR}$ (Invitrogen Corporation, Paisley, UK). Aqueous solutions were prepared with double-deionized water (Arium Pro, Sartorius AG, Göttingen, Germany). 


\section{Preparation of nanoparticles}

Formulations containing PLGA were prepared using a solvent emulsification-evaporation method based on an oil in water $(\mathrm{o} / \mathrm{w})$ single emulsion technique. ${ }^{13}$ Following the standard procedure, $200 \mathrm{mg}$ of PLGA were dissolved in $2 \mathrm{~mL}$ of ethyl acetate, and then added to $8 \mathrm{~mL}$ of a $2 \%$ (w/v) poly(vinyl alcohol) aqueous solution. The emulsion formed was homogenized using a sonicator (VibraCell VCX 130 equipped with a VC 18 probe, Sonics \& Materials Inc., Newtown, CT, USA) at 70\% amplitude for 30 seconds. The previous emulsion was then added to $15 \mathrm{~mL}$ of a $0.2 \%$ (w/v) poly(vinyl alcohol) aqueous solution and the organic solvent was removed by evaporation using a rotavapor for 90 minutes $\left(300 \mathrm{hPa}, 35^{\circ} \mathrm{C}\right)$. NPs were then recovered by centrifugation $\left(21,000 \times g, 10\right.$ minutes, $\left.4^{\circ} \mathrm{C}\right)$ and washed three times with $20 \mathrm{~mL}$ of water. After final redispersion, the NPs were transferred to $20 \mathrm{~mL}$ aluminum-sealed screw-neck vials (La-Pha-Pack ${ }^{\circledR} \mathrm{GmbH}$, Langerwehe, Germany) and stored at $4^{\circ} \mathrm{C}$ until further analysis.

The same method was used to associate MTX and SPIONs into PLGA NPs, both separate and simultaneously, by adding the previous components ( $40 \mu \mathrm{L}$ of SPIONs and/ or $20 \mathrm{mg}$ of MTX) to the organic phase. ${ }^{14}$ A schematic of the preparation process is shown in Figure 1.

\section{Conjugation of anti-CD64 antibody to nanoparticles}

Considering that CD64 is a FcyRI receptor, ${ }^{12}$ the anti-CD64 antibody should be linked through the $\mathrm{F}_{\mathrm{ab}}$ fragment, leaving the $\mathrm{F}_{\mathrm{c}}$ region available for macrophage recognition. Therefore, the coupling reaction was carried out in the presence of EDC and NHS, allowing the carboxyl-terminated NPs to react with the primary amine of the antibody present at the $\mathrm{F}_{\mathrm{ab}}$ region, and yielding an amide bond. Figure 2 summarizes the conjugation process. Following an adapted protocol, ${ }^{15}$ $10 \mathrm{~mL}$ of purified NPs were centrifuged $(21,000 \times g, 10$ minutes, $4^{\circ} \mathrm{C}$ ) and redispersed in $10 \mathrm{~mL}$ of MES buffer $(\mathrm{pH}$ 5.0). The $\mathrm{pH}$ was maintained at $5.0 \mathrm{in}$ order to maximize the attachment of EDC to the PLGA carboxyl groups. ${ }^{15}$ Activation was achieved by adding $1 \mathrm{~mL}$ of $0.1 \mathrm{M} \mathrm{EDC}$ and $1 \mathrm{~mL}$ of 0.7 M NHS (both dissolved in MES buffer, $\mathrm{pH}$ 5.0) to the NP suspension, which was kept at room temperature under moderate stirring for 1 hour. To remove the remaining reagents, the activated NPs were centrifuged $\left(21,000 \times g, 10\right.$ minutes, $\left.4^{\circ} \mathrm{C}\right)$ and redispersed in phosphate-buffered saline, yielding a final concentration of $1.0 \mathrm{mg} / \mathrm{mL}$. To conjugate the antibody to the activated NPs, $10 \mu \mathrm{L}$ of the anti-CD64 antibody solution were added to $1 \mathrm{~mL}$ of activated NP suspension. After homogenizing with a vortex mixer, the suspensions were incubated at $4^{\circ} \mathrm{C}$ for 24 hours. The conjugated NPs were again centrifuged $\left(21,000 \times g, 10\right.$ minutes, $\left.4^{\circ} \mathrm{C}\right)$ to remove excess unconjugated antibody and remaining reagents. The supernatant was stored for further antibody quantification analysis.

\section{Particle size and zeta potential measurements}

The produced NPs were characterized for particle size, size distribution (polydispersity index), and zeta potential. Mean hydrodynamic diameter and polydispersity index were assessed by dynamic light scattering using a 90 Plus particle size analyzer (Brookhaven Instruments Corporation, Holtsville, NY, USA) and zeta potential was determined by phase analysis light scattering using a ZetaPALS zeta potential analyzer (Brookhaven) at $660 \mathrm{~nm}$, with a detection angle of $90^{\circ}$ at $25^{\circ} \mathrm{C}$. All samples were diluted in water to a suitable scattering intensity and measurements were performed with three independent batches of NPs (six runs, ten cycles each).

\section{Scanning electron microscopy}

In order to evaluate the surface morphology of the NPs, scanning electron microscopy was performed using a high resolution Quanta ${ }^{\mathrm{TM}} 400$ scanning electron microscope (FEI Company, Hillsboro, OR, USA). Samples were mounted on metal stubs and coated with a gold/palladium thin film by sputtering for 60 seconds, with a $15 \mathrm{~mA}$ current, using a SPI

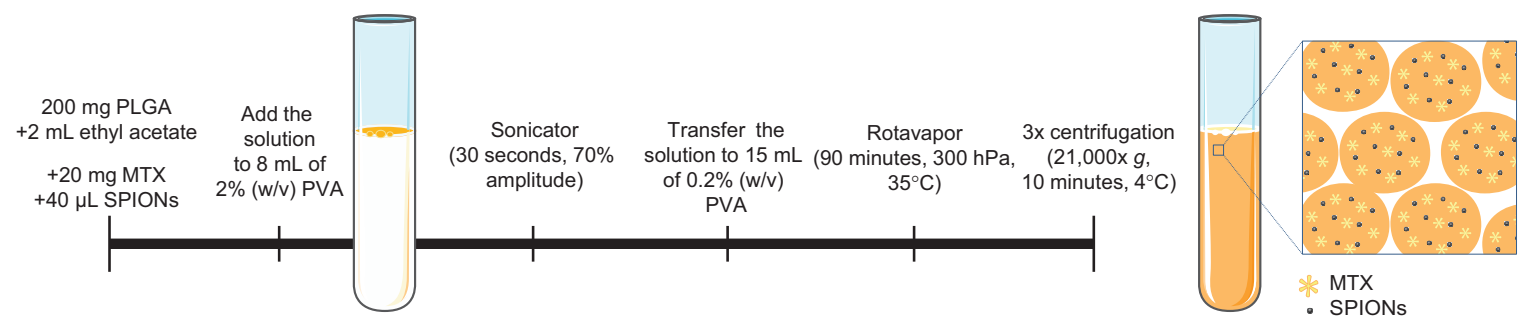

Figure I Schematic of the preparation of nanoparticles using a solvent emulsification-evaporation method based on an oil in water single emulsion technique. Abbreviations: MTX, methotrexate; SPIONs, superparamagnetic iron oxide nanoparticles; PLGA, poly(lactic-co-glycolic acid); PVA, poly(vinyl alcohol). 


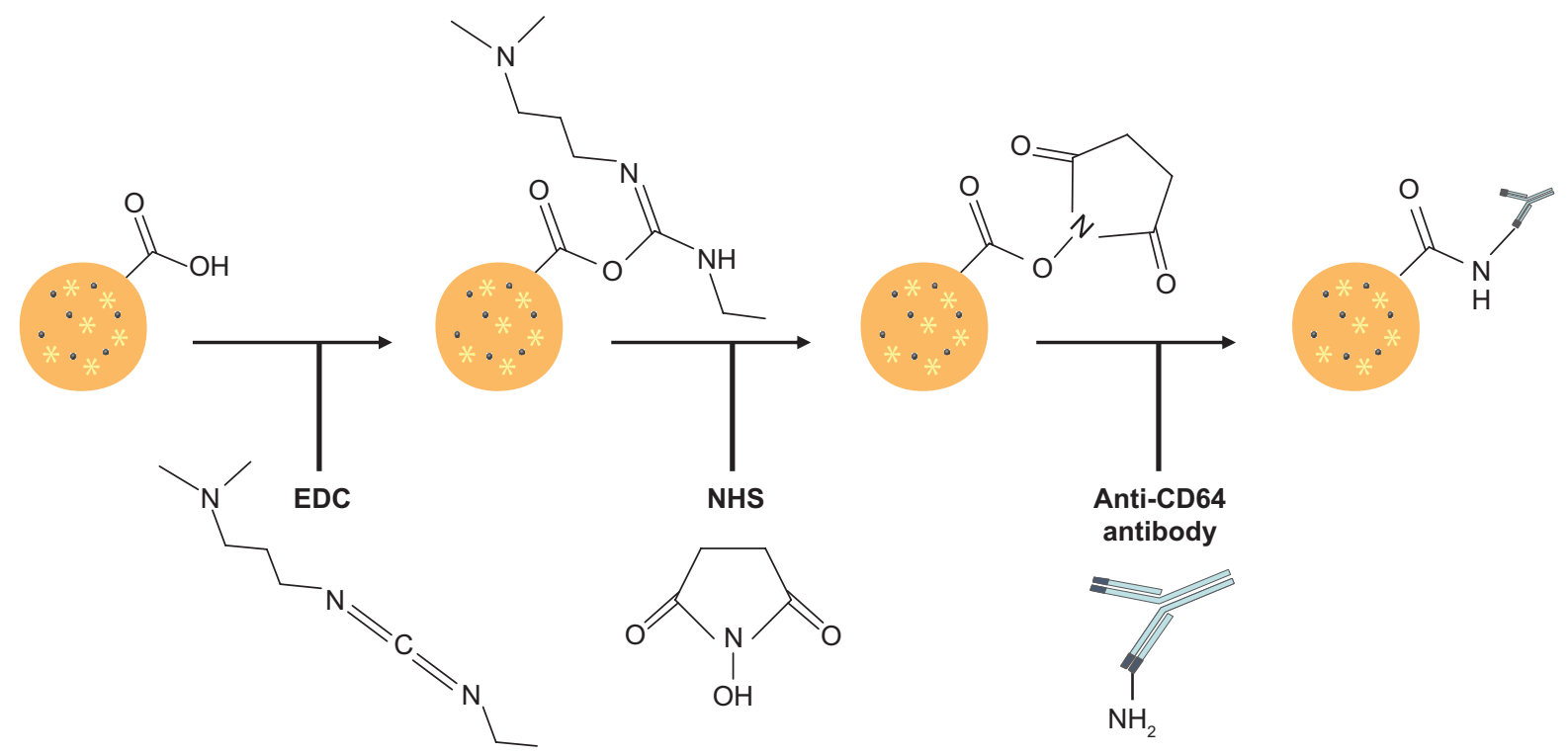

Figure 2 Schematic of the functionalization of poly(lactic-co-glycolic acid) nanoparticles with the anti-CD64 antibody.

Abbreviations: EDC, I-ethyl-3-(3-dimethylaminopropyl) carbodiimide hydrochloride; NHS, N-hydroxysulfosuccinimide.

Module sputter coater system. Images were obtained at an acceleration voltage of $15 \mathrm{kV}$.

\section{Transmission electron microscopy}

The morphological features of the developed NPs and the presence of SPIONs were assessed by transmission electron microscopy. Samples were prepared by placing $10 \mu \mathrm{L}$ of NP dispersion on a copper-mesh grid and, after 2 minutes, excess water was removed by capillarity using filter paper. For contrasting, 10 $\mu \mathrm{L}$ of $0.75 \%$ uranyl acetate solution was added and left at room temperature for 30 seconds. The grids were then observed using a JEM-1400 transmission electron microscope (JEOL Ltd., Tokyo, Japan), with an acceleration voltage of $80 \mathrm{kV}$.

\section{Methotrexate association efficiency}

The association efficiency of MTX on NPs was determined by calculating the ratio between the amount of MTX measured in the NPs and the total amount of MTX, both quantified in the NPs and in the three supernatants collected during the purification protocol, as follows:

Association efficiency $(\%)=\frac{\text { MTX in NPs }}{\text { Total amount of MTX }} \times 100 \%$

The quantification was performed by high-performance liquid chromatography (HPLC) with ultraviolet detection. The HPLC system comprised a MD-2015 multi-wavelength detector (Jasco, Easton, MD, USA) programmed for peak detection at $302 \mathrm{~nm}$, a high-pressure pump (PU-2089), an autosampler (AS-2057), and a controller (LC-Net II/ADC) mastered by ChromNAV software. A reversed-phase monolithic column Chromolith RP-18e $(100 \times 4.6 \mathrm{~mm}$ internal diameter; Merck) connected to a guard column of the same material ( $5 \times 4.6 \mathrm{~mm}$ internal diameter) was used as stationary phase. Separation conditions were adapted ${ }^{16}$ and conducted by isocratic mode (mobile phase containing $10 \%[\mathrm{v} / \mathrm{v}]$ acetonitrile and $90 \%[\mathrm{v} / \mathrm{v}]$ of $\mathrm{pH} 6.0$ phosphate/citrate buffer) at $1.0 \mathrm{~mL} / \mathrm{min}$. The phosphate/citrate buffer was composed by $0.2 \mathrm{M}$ dipotassium phosphate and $0.1 \mathrm{M}$ citric acid (63:37; $\mathrm{v} / \mathrm{v})$. The sample volume was fixed at $20 \mu \mathrm{L}$.

Standard MTX solutions were prepared at 1, 3, 6, 10, 25,50 , and $100 \mu \mathrm{g} / \mathrm{mL}$ in mobile phase. To prepare the NP samples for HPLC analyses, $100 \mu \mathrm{L}$ of NP dispersion or $100 \mu \mathrm{L}$ of supernatant were added to $900 \mu \mathrm{L}$ of mobile phase, to a final concentration of $10 \%(\mathrm{v} / \mathrm{v})$. MTX-free PLGA NPs, namely PLGA NPs and SPIONs-loaded PLGA NPs, were also analyzed and no interference was observed on the chromatograms.

\section{Anti-CD64 antibody conjugation efficiency}

The Bradford assay was performed using the Coomassie Plus ${ }^{\mathrm{TM}}$ (Bradford) assay kit to assess the efficiency of antiCD64 antibody conjugation to multifunctional NPs,${ }^{17}$ following the instructions. Diluted bovine serum albumin standards were prepared $(2.5-25 \mu \mathrm{g} / \mathrm{mL})$ using the same solvent as used for the samples (supernatant of the centrifugation of activated PLGA NPs). Coomassie Plus ${ }^{\mathrm{TM}}$ reagent was added to the supernatant of the centrifugation of anti-CD64 conjugated NPs. The protein concentration for each unknown 
sample was determined and the conjugation efficiency (\%) was assessed, as follows:

$$
\text { Conjugation efficiency }=\frac{\mathrm{A}-\mathrm{P}}{\mathrm{A}} \times 100
$$

where $\mathrm{A}$ is the initial amount of antibody and $\mathrm{P}$ is the protein in supernatant.

\section{Fourier transform infrared spectroscopy}

NPs were characterized by Fourier transform infrared spectroscopy (FT-IR), using a Frontier FT-IR spectrometer with universal attenuated total reflectance sampling accessory (PerkinElmer, Waltham, MA, USA). For each NP spectrum, a 50-scan was collected with $4 \mathrm{~cm}^{-1}$ resolution in the midinfrared region $\left(3,600-600 \mathrm{~cm}^{-1}\right)$. For these analyses, the NP dispersions were lyophilized using a VirTis AdVantage 2.0 BenchTop freeze dryer (SP Scientific, Gardiner, NY, USA). Samples were frozen at $-60^{\circ} \mathrm{C}$ for 12 hours, and primary drying was performed at $20^{\circ} \mathrm{C}$ and 150 mTorr for 20 hours. Additionally, secondary drying was performed at $25^{\circ} \mathrm{C}$ and $100 \mathrm{mTorr}$ for 20 hours, for complete sublimation. The condenser was maintained at $-80^{\circ} \mathrm{C}$ and $150 \mathrm{mTorr}$.

\section{Cell culture}

Murine macrophage RAW 264.7 cells (passages 31-38) from the American Type Culture Collection (ATCC ${ }^{\circledR}$ TIB-71 ${ }^{\mathrm{TM}}$, Rockville, MD, USA) were cultured in DMEM supplemented with $10 \%(\mathrm{v} / \mathrm{v})$ fetal bovine serum, $1 \%(\mathrm{v} / \mathrm{v})$ penicillin-streptomycin, and 1\% (v/v) Fungizone ${ }^{\circledR}$ antimycotic. The cells were maintained in a humidified chamber at $37^{\circ} \mathrm{C}$ and $5 \% \mathrm{CO}_{2}$, and the culture medium was changed every $2-3$ days. Cells were detached using a scraper and harvested at $80 \%$ confluence.

\section{Effect of nanoparticles on cell viability and cytotoxicity}

Following exposure to the developed NPs, MTT and LDH assays were performed to measure cell viability and cytotoxicity, respectively. ${ }^{18}$ Briefly, RAW 264.7 cells were cultured in 96-well plates at a density of $2.5 \times 10^{4}$ cells $/ \mathrm{mL}$ and cultured for 24 hours before use. The following day, the culture medium was removed, and the NP dispersions or free MTX were added at different concentrations (corresponding to $0.01-100 \mu \mathrm{g} / \mathrm{mL}$ MTX). MTX-free NPs were added at concentrations corresponding to the polymer concentration of the MTX-loaded NPs (approximately $0.15-1,500 \mu \mathrm{g} / \mathrm{mL}$ ). Two controls, ie, cells treated with culture medium and cells treated with Triton ${ }^{\mathrm{TM}}$ $\mathrm{X}-1002 \%(\mathrm{w} / \mathrm{v})$ in culture medium, were also included.

For the MTT assay, ${ }^{18}$ after 24 hours of incubation, the culture medium was removed and replaced by $200 \mu \mathrm{L}$ of MTT diluted in fresh DMEM at $0.5 \mathrm{mg} / \mathrm{mL}$. The plate was incubated for 4 hours at $37^{\circ} \mathrm{C}$ in the dark. The MTT solution was discarded and formazan crystals were solubilized using 200 $\mu \mathrm{L}$ of dimethyl sulfoxide. The plate was shaken for $10 \mathrm{~min}-$ utes at room temperature, and absorbance (590 nm, $630 \mathrm{~nm}$ ) was measured using a Synergy ${ }^{\mathrm{TM}}$ HT Multi-mode microplate reader (BioTek Instruments Inc., Winooski, VT, USA).

The LDH assay was performed following the instructions. Briefly, after 24 hours of incubation, the plate was centrifuged (250× $g, 10$ minutes, at room temperature) and $100 \mu \mathrm{L}$ were collected and transferred to a new 96-well plate. The LDH cytotoxicity detection kit reaction mixture was added, and absorbance (490 nm, $630 \mathrm{~nm}$ ) was read after 20 minutes of incubation at room temperature in the dark. Cell viability and cytotoxicity were assessed and expressed as a percentage in relation to both controls.

\section{Statistical analysis}

Statistical analysis was performed using IBM $^{\circledR}$ SPSS $^{\circledR}$ Statistics version 21.0 (IBM Corporation, Armonk, NY, USA). The results are reported as the mean \pm standard deviation for a minimum of three independent experiments. The twotailed Student's $t$-test and one-way analysis of variance were performed to compare two or multiple independent groups, respectively. When the group was significantly different $(P<0.01)$, differences between groups were compared with the Tukey's post hoc test. Paired samples were analyzed with the paired-samples two-tailed Student's $t$-test. Differences were considered to be statistically significant at $P<0.01$.

\section{Results and discussion}

For a successful RA-targeted theranostic approach, it was paramount that all components in the devised PLGA NPs, ie, SPIONs (for imaging diagnosis), MTX (therapeutic drug), and the anti-CD64 antibody (for specific RA macrophage targeting), were effectively integrated in the nanoparticulate system, without significantly altering their known drug delivery characteristics.

The NPs were designed as part of an intravenous administration strategy for RA-targeted therapy and imaging. Therefore, the physicochemical properties of the developed NPs, which influence their physical stability and interaction with biological tissues, deserved detailed attention. The NPs were characterized in terms of their particle size, polydispersity index, zeta potential, association with SPIONs and MTX, anti-CD64 antibody conjugation, and their effect on cell viability and cytotoxicity.

The mean particle size of the developed NPs, measured by dynamic light scattering, is presented in Table 1. All 
Table I Physicochemical properties of the developed multifunctional nanoparticles

\begin{tabular}{|c|c|c|c|c|c|c|}
\hline Formulation & $\begin{array}{l}\text { Mean effective } \\
\text { diameter } \\
(\mathrm{nm})\end{array}$ & PdI & $\begin{array}{l}\text { Zeta } \\
\text { potential } \\
(\mathrm{mV})\end{array}$ & $\begin{array}{l}\text { MTX } \\
\text { association } \\
\text { efficiency (\%) }\end{array}$ & $\begin{array}{l}\text { Anti-CD64/ } \\
\text { NPs ratio } \\
(\mu \mathrm{g} / \mathrm{mg})\end{array}$ & $\begin{array}{l}\text { Anti-CD64 } \\
\text { conjugation } \\
\text { efficiency }(\%)\end{array}$ \\
\hline PLGA NPs & $141 \pm 18$ & $0.21 \pm 0.10$ & $-29.4 \pm 5.5$ & - & - & - \\
\hline SPIONs-loaded PLGA NPs & $|3| \pm 12$ & $0.22 \pm 0.04$ & $-25.2 \pm 5.0$ & - & - & - \\
\hline MTX-loaded PLGA NPs & $137 \pm 6.9$ & $0.23 \pm 0.10$ & $-27.3 \pm 4.4$ & $79.1 \pm 3.7$ & - & - \\
\hline $\begin{array}{l}\text { SPIONs- and MTX-loaded } \\
\text { PLGA NPs }\end{array}$ & $157 \pm 22$ & $0.11 \pm 0.10$ & $-32.5 \pm 4.6$ & $75.5 \pm 4.3$ & - & - \\
\hline $\begin{array}{l}\text { Anti-CD64-conjugated } \\
\text { PLGA NPs }\end{array}$ & $167 \pm 15^{c}$ & $0.24 \pm 0.05^{c}$ & $-18.0 \pm 4.3^{c}$ & - & $3.11 \pm 0.19$ & $31.1 \pm 1.9$ \\
\hline $\begin{array}{l}\text { Anti-CD64-conjugated } \\
\text { SPIONs-loaded PLGA NPs }\end{array}$ & $192 \pm 12^{b, c}$ & $0.15 \pm 0.06^{\mathrm{b}, \mathrm{c}}$ & $-19.3 \pm 3.0^{b, c}$ & - & $3.59 \pm 0.19$ & $35.9 \pm 1.9$ \\
\hline $\begin{array}{l}\text { Anti-CD64-conjugated } \\
\text { MTX-loaded PLGA NPs }\end{array}$ & $162 \pm 24^{c}$ & $0.25 \pm 0.11^{c}$ & $-16.4 \pm 3.2^{c}$ & $79.1 \pm 3.7$ & $3.33 \pm 0.32$ & $33.3 \pm 3.2$ \\
\hline $\begin{array}{l}\text { Anti-CD64-conjugated } \\
\text { SPIONs- and MTX-loaded } \\
\text { PLGA NPs }\end{array}$ & $202 \pm 10^{b, c}$ & $0.34 \pm 0.02^{b, c}$ & $-2 I . I \pm 2.2^{b, c}$ & $75.5 \pm 4.3$ & $3.66 \pm 0.54$ & $36.6 \pm 5.4$ \\
\hline
\end{tabular}

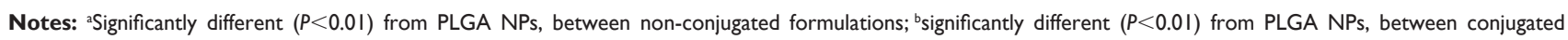
formulations; 'significantly different $(P<0.0 \mathrm{l})$ before and after functionalization. Values represent the mean \pm standard deviation ( $\mathrm{n} \geq 3$ ).

Abbreviations: MTX, methotrexate; NPs, nanoparticles; SPIONs, superparamagnetic iron oxide nanoparticles; PLGA, poly(lactic-co-glycolic acid); Pdl, polydispersity index.

formulations tested in this work were within the size range of 130-200 nm, and showed a relatively homogeneous size distribution as revealed by polydispersity index values. In order to avoid sequestration of NPs in spleen sinusoids and liver fenestrae, the size of a nanosystem should not exceed $200 \mathrm{~nm}$. Further, NPs with a diameter smaller than $6 \mathrm{~nm}$ can be excreted by the kidneys, so are rapidly eliminated from the bloodstream. ${ }^{19}$ Consequently, the sizes obtained for the developed NPs are suitable for intravenous administration. Focusing on the non-conjugated NPs, SPIONs and MTX association did not significantly affect particle size for any of the formulations, suggesting that they do not considerably interfere with NP formation, and that it is possible to create a complex multifunctional nanoparticulate system maintaining the primary properties PLGA NPs. However, conjugation with the anti-CD64 antibody interfered slightly with particle size. NPs underwent a shift in mean particle size, increasing in the order of 30-50 nm (Table 1), which could be explained by the presence of the antibody on the surface of the particles, as well as by a higher water content on hydration of the conjugate. ${ }^{20}$ The polydispersity indexes obtained were between 0.1 and 0.3 (Table 1), indicating that well defined and monodispersed nanoparticulate populations were produced with uniform and consistent sizes, and without suffering aggregation.

Regarding surface charge, all formulations tested had markedly negative zeta potential values (Table 1). A negative charge is typical of carboxyl-terminated NPs owing to the contribution of the carboxyl groups, which are deprotonated at physiological $\mathrm{pH}$ or, in this particular case, at the $\mathrm{pH}$ of double-deionized water. ${ }^{21}$ Zeta potential values around $-30 \mathrm{mV}$ contribute to the stability of hydrophobic particles in aqueous dispersion, avoiding formation of aggregates. ${ }^{22}$ The zeta potential values decreased significantly in all formulations after conjugation of anti-CD64 (Table 1). The principle behind the antibody conjugation relies on establishment of a covalent amide bond between the amine and carboxyl termini of the antibody and PLGA, respectively. Consequently, it is expected that partial surface charge shielding occurs due to the depletion of carboxyl groups at the surface as they were involved in the reaction with the amine termini of the antibody.

Scanning electron micrographs allowed gathering information about the surface morphology of the NPs. The images show the spherical shape of the NPs as well as their smooth surface, which is devoid of pores (Figure 3 ). The NPs had sizes below $200 \mathrm{~nm}$, confirming the results previously obtained by dynamic light scattering. The homogenous and flat surface, not varying between different formulations, suggests that both MTX and SPIONs are entrapped within the PLGA polymeric matrix. Images of the anti-CD64-conjugated PLGA NPs show a more aggregated and gel-like state, possibly due to the functionalization protocol and the presence of the antibody on the NP surface (Figure 3Aii, Bii, Cii and Dii).

Transmission electron micrographs (Figure 4) allowed further confirmation of the results obtained by dynamic light scattering and scanning electron microscopy with regard to particle size. The micrographs show a monodispersed population of individual, smooth, and spherical particles with well defined sizes. Association with MTX and SPIONs, as well as anti-CD64 antibody conjugation, did not considerably affect particle shape or overall size.

Figure 4Bi-Bii shows SPIONs-loaded NPs in which the SPIONs are evident inside the PLGA NPs as smaller and 
electronically denser, well dispersed spots, confirming their efficient association, both alone and when coassociated with MTX (Figure 4Di-Dii). Figure 4Aii, Bii, Cii and Dii shows micrographs of the NP formulations after conjugation with the anti-CD64 antibody. A denser and thicker "corona" is apparent surrounding the lighter NP core (Figure 4Aii), which may indicate the presence of the antibody on the surface of the NP. Similar observations using transmission electron microscopy were reported by Thamake et al for similar antibody-conjugated PLGA NPs. ${ }^{23}$
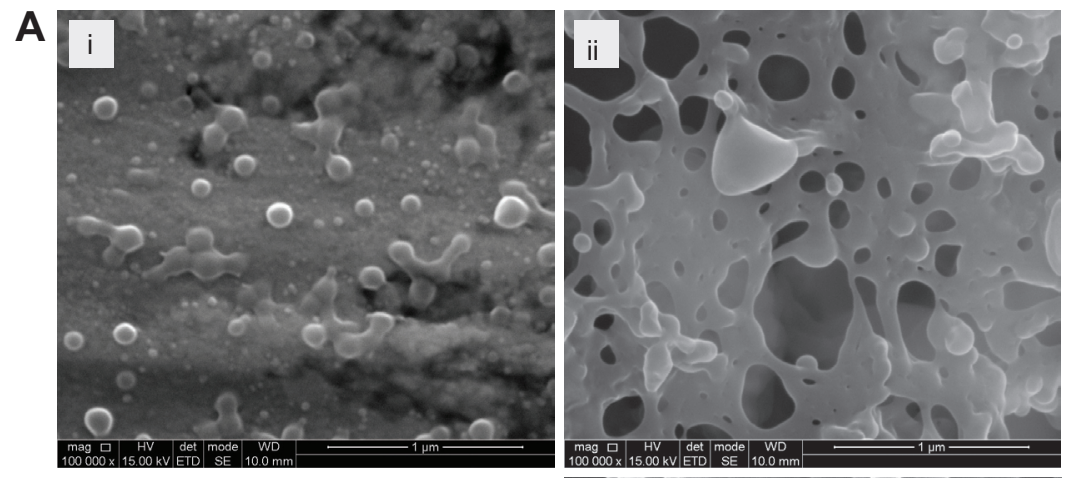

B
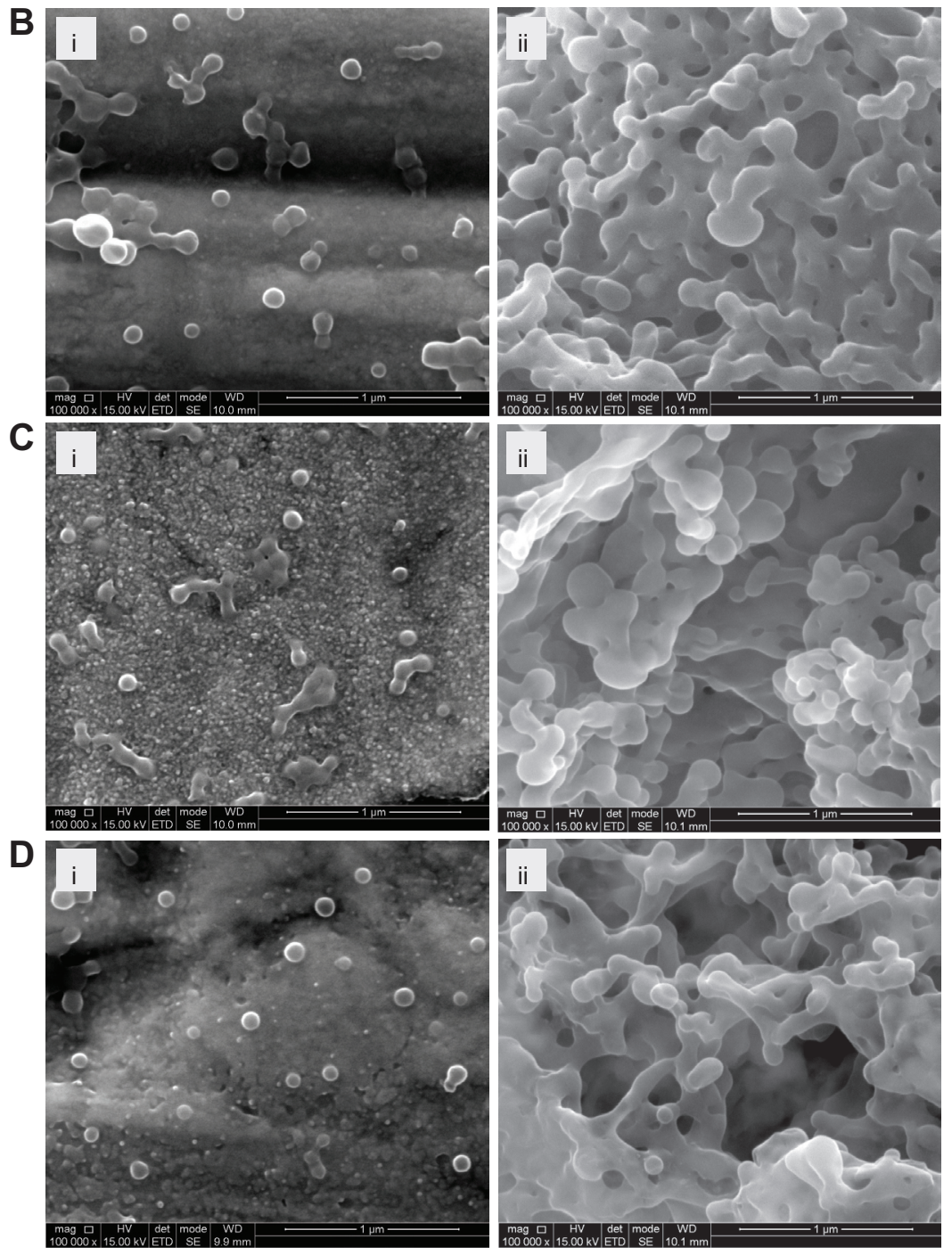

Figure 3 Scanning electron micrographs of (Ai) PLGA NPs, (Aii) anti-CD64-conjugated PLGA NPs, (Bi) SPIONs-loaded PLGA NPs, (Bii) anti-CD64-conjugated SPIONsloaded PLGA NPs, (Ci) MTX-loaded PLGA NPs, (Cii) anti-CD64-conjugated MTX-loaded PLGA NPs, (Di) SPIONs- and MTX-loaded PLGA NPs, and (Dii) anti-CD64conjugated SPIONs- and MTX-loaded PLGA NPs.

Notes: Scale bars correspond to I $\mu \mathrm{m}$. Magnification 100,000x.

Abbreviations: MTX, methotrexate; NPs, nanoparticles; SPIONs, superparamagnetic iron oxide nanoparticles; PLGA, poly(lactic-co-glycolic acid). 
A

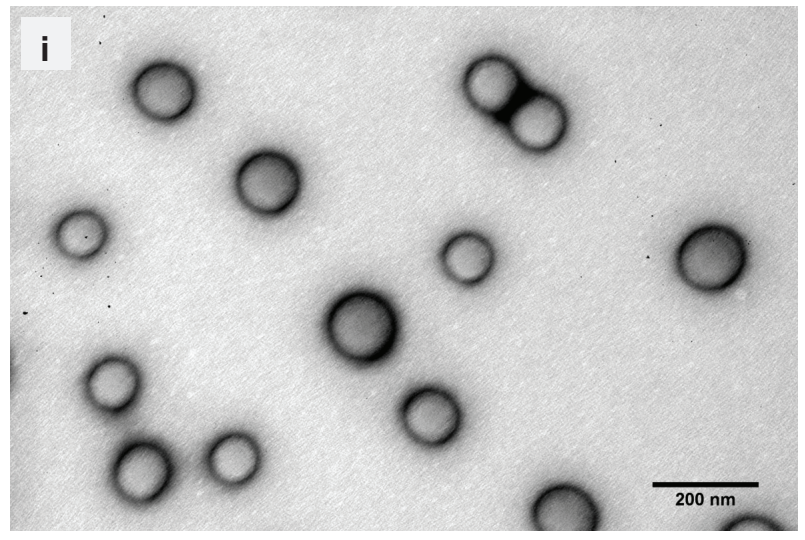

B

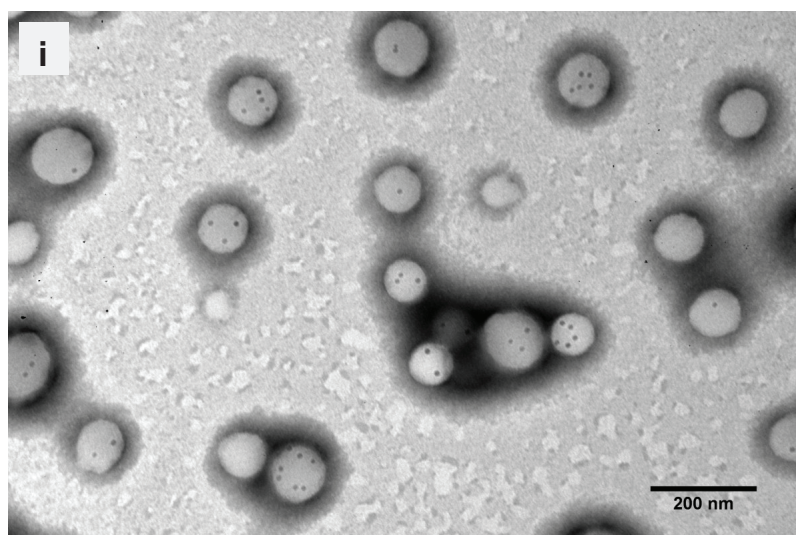

C

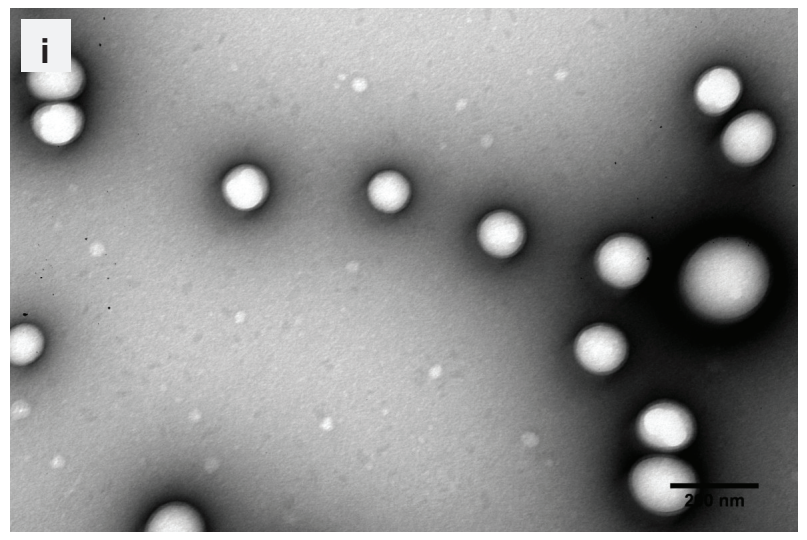

D

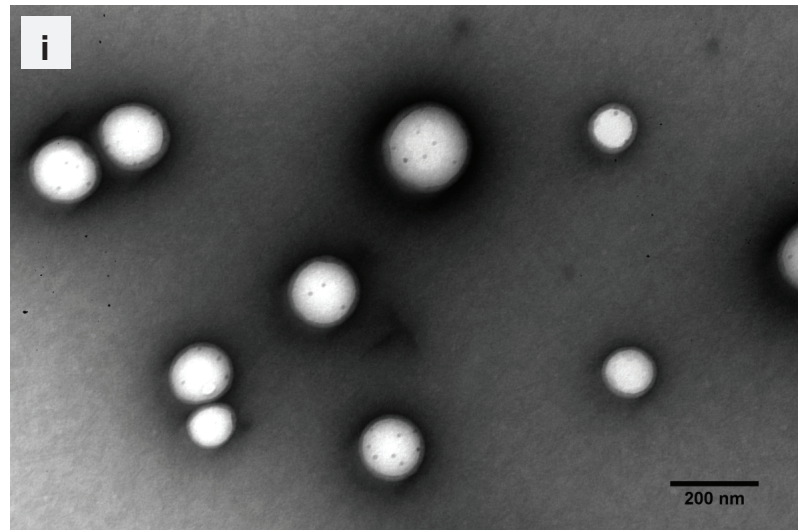

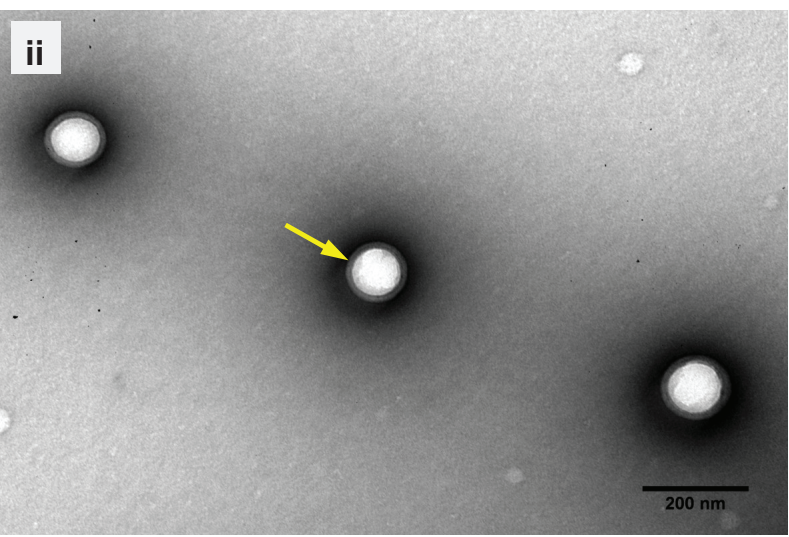
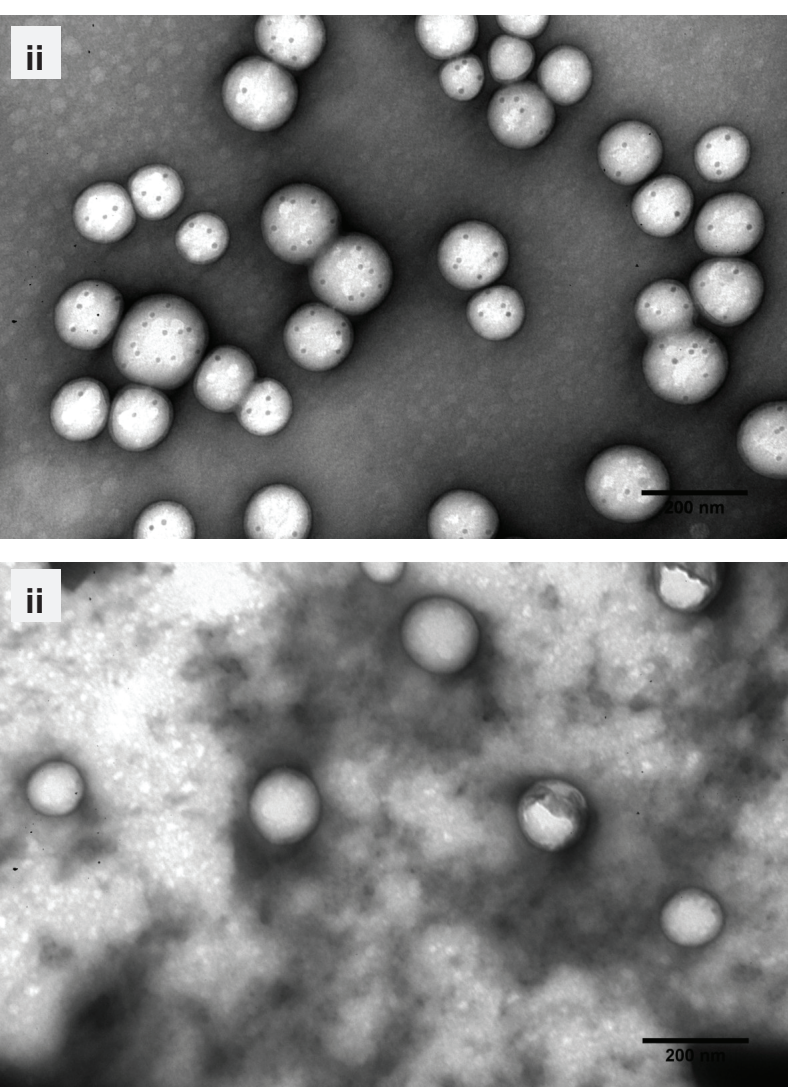

ii
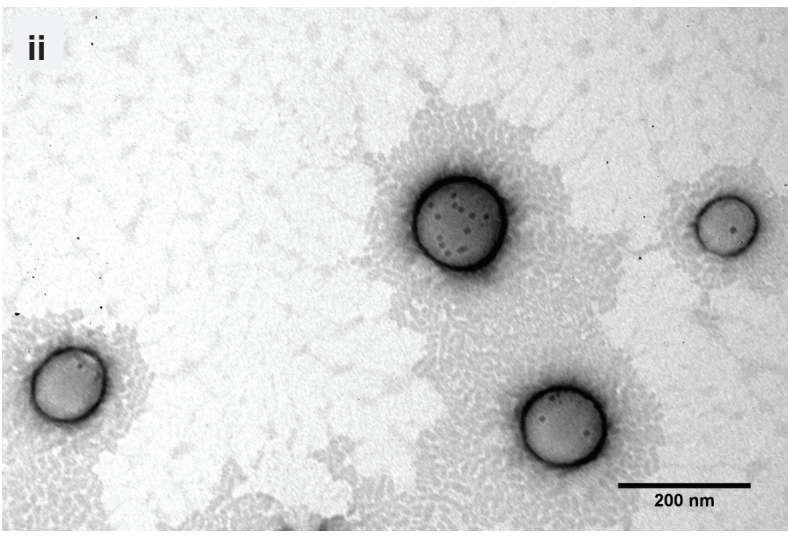

Figure 4 Transmission electron micrographs of (Ai) PLGA NPs, (Aii) anti-CD64-conjugated PLGA NPs, (Bi) SPIONs-loaded PLGA NPs, (Bii) anti-CD64-conjugated SPIONs-loaded PLGA NPs, (Ci) MTX-loaded PLGA NPs, (Cii) anti-CD64-conjugated MTX-loaded PLGA NPs, (Di) SPIONs- and MTX-loaded PLGA NPs, and (Dii) anti-CD64-conjugated SPIONs- and MTX-loaded PLGA NPs.

Notes: Scale bars correspond to $200 \mathrm{~nm}$. In (Aii) it is possible to detect a "corona"-like structure typical of surface antibody conjugation (arrow). Abbreviations: MTX, methotrexate; NPs, nanoparticles; SPIONs, superparamagnetic iron oxide nanoparticles; PLGA, poly(lactic-co-glycolic acid). 
A previously described HPLC method was used to quantify the association of MTX in the devised NPs. ${ }^{16}$ Given that MTX has a solubility of $0.01 \mathrm{mg} / \mathrm{mL}$ in water at $20^{\circ} \mathrm{C},{ }^{8}$ the MTX-loaded PLGA NPs were prepared using a single emulsion technique in order to achieve elevated values of association efficiency. High efficiency was demonstrated for both MTX-loaded and MTX- and SPIONs-loaded NPs, being $79.1 \%$ and $75.5 \%$, respectively (Table 1 ). These values did not differ significantly between the two different formulations $(P=0.32)$, indicating that a co-association of both agents is possible in a PLGA-based theranostic approach. MTX is a very effective drug against RA but is extremely toxic and has serious side effects, limiting the dose that can be administered, thereby compromising RA therapy. ${ }^{3,8}$ A high association between MTX and PLGA in a targeted nanosystem could provide a new opportunity for RA therapy, since the bioavailability, safety, and efficacy of MTX would be improved.

The anti-CD64 conjugation efficiency of each formulation is shown in Table 1. The amount of anti-CD64 present in the nanoparticulate system was shown to be approximately $3.5 \mu \mathrm{g}$ per mg of NPs. Different antibody/NPs ratios were obtained in previous works when conjugating PLGA NPs with different monoclonal antibodies. ${ }^{15,21,24}$ However, the conjugation efficiency obtained in this work was significantly higher $(31 \%-37 \%)$, because considerably lower amounts of antibody (at least 20 -fold less) were used for functionalization of the PLGA NPs. Statistical analysis of the conjugation efficiencies did not show significant differences between the

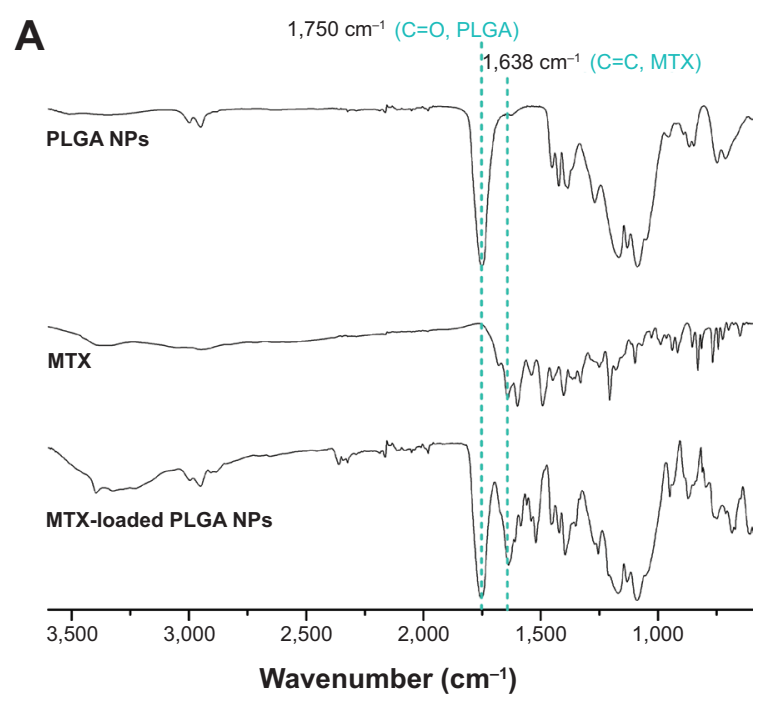

formulations ( $P=0.26$ ), indicating that association with both MTX and SPIONs did not considerably affect the main features of the NPs and their interaction with the antibody.

FT-IR spectra of the samples were obtained to confirm the association of MTX into PLGA NPs and the functionalization of the NPs with the anti-CD64 antibody. The FT-IR spectra of PLGA NPs and MTX-loaded PLGA NPs were compared to the FT-IR spectrum of free MTX (Figure 5A). At 1,750 $\mathrm{cm}^{-1}$, a marked peak indicates the presence of a carbonyl bond $(\mathrm{C}=\mathrm{O}$ stretching vibration), which is characteristic of PLGA. ${ }^{25}$ Further, in the MTX spectrum, it is possible to observe a different peak at $1,638 \mathrm{~cm}^{-1}(\mathrm{C}=\mathrm{C}$ stretching vibration), which is characteristic of the drug molecule but not of PLGA. ${ }^{26}$ The characteristic peak from PLGA was not altered in the MTX-loaded NPs spectrum, and the carbon-carbon double bond typical of MTX is evident in the spectrum, confirming that MTX was successfully associated into the PLGA NPs.

The FT-IR spectra of PLGA NPs and anti-CD64 conjugated PLGA NPs were compared with the spectrum of the anti-CD64 antibody (Figure 5B). At $1,750 \mathrm{~cm}^{-1}$, the PLGA-characteristic peak is also present in the antibody ( $\mathrm{C}=\mathrm{O}$ stretching bond). ${ }^{25} \mathrm{Near} 1,640 \mathrm{~cm}^{-1}$, an amide bond ( $\mathrm{C}=\mathrm{N}$ stretching vibration) should be recognized, identifying conjugation of the antibody to PLGA NPs. However, at this wavenumber, both PLGA NPs and anti-CD64 antibody spectra show again a clear peak, which corresponds to the $\mathrm{C}=\mathrm{O}$ stretching bond present on both components, not allowing us to immediately draw conclusions regarding conjugation

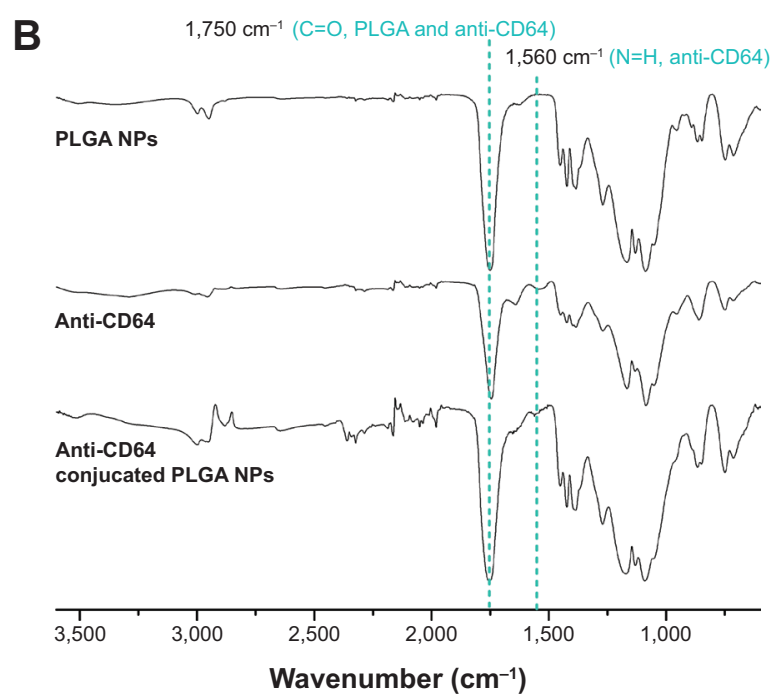

Figure 5 (A) FT-IR spectra of PLGA NPs, free MTX, and MTX-loaded PLGA NPs. (B) FT-IR spectra of PLGA NPs, free anti-CD64 antibody, and anti-CD64-conjugated PLGA NPs.

Abbreviations: MTX, methotrexate; NPs, nanoparticles; PLGA, poly(lactic-co-glycolic acid); FT-IR, Fourier transform infrared. 
of the NPs. ${ }^{26,27}$ Despite this, in the anti-CD64 spectrum, an additional peak at $1,560 \mathrm{~cm}^{-1}$ stands out, corresponding to the amine groups of the antibody (N-H bending vibrations). ${ }^{27}$ In the case of the anti-CD64 conjugated NPs spectrum, this characteristic peak emerges, confirming that anti-CD64 was present in the functionalized PLGA NPs, either covalently linked or physically adsorbed.

The effect of NPs on cell viability and cytotoxicity after 24 hours of incubation was studied in vitro on RAW 264.7 cells, performing MTT and LDH assays, respectively, as a function of the devised formulations and the different concentrations of MTX $(0.01-100 \mu \mathrm{g} / \mathrm{mL})$. Both MTT and LDH assays reveal the same tendency and allow similar conclusions to be drawn. All formulations displayed a concentration-dependent effect, with toxicity increasing proportional to the concentration (Figure 6). The results also demonstrated that the toxicity of MTXloaded NPs was greater than of the free drug, suggesting that a future approach for RA therapy based on these NPs may enhance the therapeutic efficiency of MTX. Additionally, with the exception of the highest concentration,
MTX-free NPs did not significantly affect cell viability, confirming the safety profile of the devised nanosystem (Figure 6).

Anti-CD64-conjugated NPs did not originate higher cytotoxicity when compared with non-conjugated NPs. This is justified by the fact that RAW 264.7 macrophages, being a mouse cell line, do not express the human CD64 receptor, and these cells were used in this assay as a cell model that does not express this receptor. In Figure 6, it is apparent that anti-CD64-conjugated MTX-loaded NPs are not as toxic as non-conjugated MTX-loaded NPs, demonstrating that this system may work as a targeted approach.

Future work using cell models that express or are modified for overexpressing the CD64 receptor will allow studying of the targeting ability of the anti-CD64-conjugated NPs, aiming for the envisioned theranostic application.

In this work, MTX, SPIONs, and anti-CD64 antibody were successfully co-associated into PLGA NPs for the management of RA. The physicochemical features of the devised NPs, ie, their size, zeta potential, morphology, high MTX association efficiency, association with SPIONs, anti-CD64

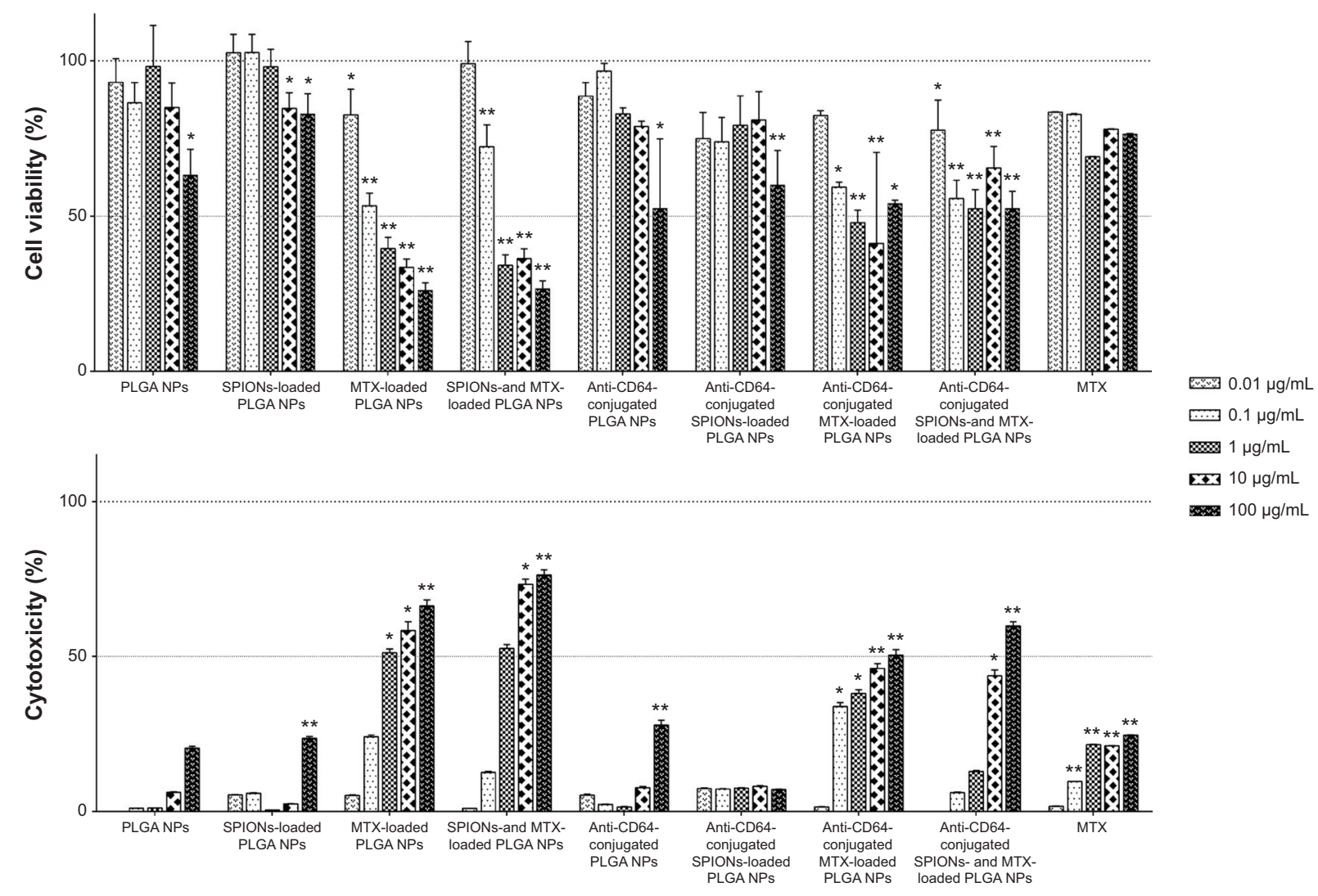

Figure 6 Cytotoxicity and effect on the viability of RAW 264.7 cells as a function of the different NP formulations and concentrations of MTX ( 0.01 , 0.1 , I, I0, and I00 $\mu$ g/mL) tested.

Notes: MTX-free NP concentrations correspond to the polymer concentration of MTX-loaded NPs. Values represent the mean \pm standard deviation ( $\geq 3$; $* P<0.05$; $* * P<0.01)$

Abbreviations: MTX, methotrexate; NPs, nanoparticles; PLGA, poly(lactic-co-glycolic acid); SPIONs, superparamagnetic iron oxide nanoparticles. 
functionalization, and in vitro safety profile are key elements for a future biomedical and pharmaceutical approach. This new design for a targeted RA theranostic strategy could be considered and studied in order to find new means for RA therapy and also work as an enhanced imaging tool for techniques such as MRI.

\section{Conclusion}

Multifunctional PLGA-based nanocarriers for drug targeting and in vivo imaging are of particular interest due to their biodegradability and biocompatibility. In this work, by effectively co-associating MTX and SPIONs into PLGA NPs, and successfully functionalizing them with an anti-CD64 antibody, a novel attempt was made to achieve targeted therapy and imaging for RA.

Overall, the association of both MTX and SPIONs did not significantly affect the properties of the PLGA NPs. The NPs had a reduced particle size and were stable in solution, which are paramount requisites for their application as drug delivery systems. Consequently, the proposed nanoparticulate system may potentiate the action of MTX without injuring healthy tissues and organs, simultaneously providing a non-invasive and specific imaging tool for RA. After their development and thorough characterization in this study, these NPs are now ready for further in vitro studies aiming for the assessment of their performance in targeting RA macrophages and reducing inflammation at sites of RA.

\section{Acknowledgments}

The authors would like to acknowledge Purac Biomaterials, Ocean NanoTech, and Excella for kindly providing the PLGA, SPIONs, and MTX, respectively. The authors are also grateful to Dr Daniela Silva (Centro de Materiais da Universidade do Porto [CEMUP], University of Porto) and Dr Rui Fernandes (Histology and Electron Microscopy Service - Instituto de Biologia Molecular e Celular [HEMS-IBMC], University of Porto) for their expertise with scanning and transmission electron microscopy. This work was financed by the European Regional Development Fund (ERDF) through the Programa Operacional Factores de Competitividade (COMPETE), by Portuguese funds through FCT in the framework of the PEst-C/SAU/LA0002/2013 and PEst-C/EQB/LA0006/2013 projects, and was cofinanced by the North Portugal Regional Operational Programme (ON.2, O Novo Norte) within the framework of the SAESCTN-PIIC\&DT/2011 project, under the National Strategic Reference Framework (NSRF), and project NORTE-07-0124-FEDER-000067.

\section{Disclosure}

The authors state that the current paper includes work based on the master's thesis submitted by CCM as partial fulfillment of the requirements to conclude her degree in bioengineering at the Faculty of Engineering, University of Porto. Otherwise, the authors report no conflicts of interest in this work.

\section{References}

1. Davis JM III, Matteson EL. My treatment approach to rheumatoid arthritis. Mayo Clin Proc. 2012;87(7):659-673.

2. Firestein GS. Evolving concepts of rheumatoid arthritis. Nature. 2003; 423(6937):356-361

3. Pope RM. Apoptosis as a therapeutic tool in rheumatoid arthritis. Nat Rev Cancer. 2002;2(7):527-535.

4. Vonkeman HE, van de Laar MA. The new European League Against Rheumatism/American College of Rheumatology diagnostic criteria for rheumatoid arthritis: how are they performing? Curr Opin Rheumatol. 2013;25(3):354-359.

5. Ragheb RRT, Kim D, Bandyopadhyay A, et al. Induced clustered nanoconfinement of superparamagnetic iron oxide in biodegradable nanoparticles enhances transverse relaxivity for targeted theranostics. Magn Reson Med. 2013;70(6):1748-1760.

6. Stephen ZR, Kievit FM, Zhang M. Magnetite nanoparticles for medical MR imaging. Mater Today. 2011;14(7-8):330-338.

7. Rosen JE, Chan L, Shieh DB, Gu FX. Iron oxide nanoparticles for targeted cancer imaging and diagnostics. Nanomedicine. 2012;8(3):275-290.

8. Abolmaali SS, Tamaddon AM, Dinarvand R. A review of therapeutic challenges and achievements of methotrexate delivery systems for treatment of cancer and rheumatoid arthritis. Cancer Chemother Pharmacol. 2013;71(5):1115-1130.

9. Smolen JS, Landewe R, Breedveld FC, et al. EULAR recommendations for the management of rheumatoid arthritis with synthetic and biological disease-modifying antirheumatic drugs: 2013 update. Ann Rheum Dis. 2014;73(3):492-509.

10. Ferrari M. Cancer nanotechnology: opportunities and challenges. Nat Rev Cancer. 2005;5(3):161-171.

11. Panyam J, Labhasetwar V. Biodegradable nanoparticles for drug and gene delivery to cells and tissue. Adv Drug Deliv Rev. 2003;55(3): 329-347.

12. van Roon JA, van Vuuren AJ, Wijngaarden S, et al. Selective elimination of synovial inflammatory macrophages in rheumatoid arthritis by an Fc-gamma receptor I-directed immunotoxin. Arthritis Rheum. 2003; 48(5):1229-1238.

13. Sarmento B, Martins S, Ferreira D, Souto EB. Oral insulin delivery by means of solid lipid nanoparticles. Int J Nanomedicine. 2007;2(4):743-749.

14. Niu C, Wang Z, Lu G, et al. Doxorubicin loaded superparamagnetic PLGA-iron oxide multifunctional microbubbles for dual-mode US/MR imaging and therapy of metastasis in lymph nodes. Biomaterials. 2013; 34(9):2307-2317.

15. Scott CJ, Marouf WM, Quinn DJ, et al. Immunocolloidal targeting of the endocytotic siglec-7 receptor using peripheral attachment of siglec-7 antibodies to poly(lactide-co-glycolide) nanoparticles. Pharm Res. 2008;25(1):135-146.

16. United States Pharmacopeial Convention, Methotrexate. Revision Bulletin, Official May 1, 2010. Available from: http://www.usp.org/sites/default/ files/usp_pdf/EN/USPNF/methotrexate.pdf. Accessed August 31, 2014.

17. Bradford MM. A rapid and sensitive method for the quantitation of microgram quantities of protein utilizing the principle of protein-dye binding. Anal Biochem. 1976;72(1-2):248-254.

18. Mosmann T. Rapid colorimetric assay for cellular growth and survival: application to proliferation and cytotoxicity assays. J Immunol Methods. 1983;65(1-2):55-63. 
19. Albanese A, Tang PS, Chan WC. The effect of nanoparticle size, shape, and surface chemistry on biological systems. Annu Rev Biomed Eng. 2012;14:1-16.

20. Xu H, Aguilar ZP, Yang L, et al. Antibody conjugated magnetic iron oxide nanoparticles for cancer cell separation in fresh whole blood. Biomaterials. 2011;32(36):9758-9765.

21. Kocbek P, Obermajer N, Cegnar M, Kos J, Kristl J. Targeting cancer cells using PLGA nanoparticles surface modified with monoclonal antibody. J Control Release. 2007;120(1-2):18-26.

22. Muller RH, Jacobs C, Kayser O. Nanosuspensions as particulate drug formulations in therapy. Rationale for development and what we can expect for the future. Adv Drug Deliv Rev. 2001;47(1):3-19.

23. Thamake SI, Raut SL, Ranjan AP, Gryczynski Z, Vishwanatha JK. Surface functionalization of PLGA nanoparticles by non-covalent insertion of a homo-bifunctional spacer for active targeting in cancer therapy. Nanotechnology. 2011;22(3):035101.
24. Cirstoiu-Hapca A, Bossy-Nobs L, Buchegger F, Gurny R, Delie F. Differential tumor cell targeting of anti-HER2 (Herceptin) and antiCD20 (Mabthera) coupled nanoparticles. Int J Nanomedicine. 2007; 331(2):190-196.

25. Choi S-W, Kim J-H. Design of surface-modified poly(d,1-lactide-coglycolide) nanoparticles for targeted drug delivery to bone. J Control Release. 2007;122(1):24-30.

26. Kohler N, Sun C, Wang J, Zhang M. Methotrexate-modified superparamagnetic nanoparticles and their intracellular uptake into human cancer cells. Langmuir. 2005;21(19):8858-8864.

27. Sarmento B, Ferreira D, Veiga F, Ribeiro A. Characterization of insulinloaded alginate nanoparticles produced by ionotropic pre-gelation through DSC and FTIR studies. Carbohydr Polym. 2006;66(1):1-7.
International Journal of Nanomedicine

\section{Publish your work in this journal}

The International Journal of Nanomedicine is an international, peerreviewed journal focusing on the application of nanotechnology in diagnostics, therapeutics, and drug delivery systems throughout the biomedical field. This journal is indexed on PubMed Central, MedLine, CAS, SciSearch ${ }^{\circledR}$, Current Contents ${ }^{\circledR} /$ Clinical Medicine,

\section{Dovepress}

Journal Citation Reports/Science Edition, EMBase, Scopus and the Elsevier Bibliographic databases. The manuscript management system is completely online and includes a very quick and fair peer-review system, which is all easy to use. Visit http://www.dovepress.com/ testimonials.php to read real quotes from published authors. 